\title{
Effects of meditation experience on functional connectivity of distributed brain networks
}

\section{Wendy Hasenkamp* and Lawrence W. Barsalou}

Department of Psychology, Emory University, Atlanta, GA, USA

Edited by:

Amishi P. Jha, University of Miami, USA

\section{Reviewed by:}

Juliana Yordanova, Bulgarian Academy of Sciences, Bulgaria

Rebecca Elliott, University of

Manchester, UK

*Correspondence:

Wendy Hasenkamp, Department of

Psychology, Emory University, 36

Eagle Row, Suite 270, Atlanta, GA

30322, USA.

e-mail:whasenk@emory.edu
This study sought to examine the effect of meditation experience on brain networks underlying cognitive actions employed during contemplative practice. In a previous study, we proposed a basic model of naturalistic cognitive fluctuations that occur during the practice of focused attention meditation. This model specifies four intervals in a cognitive cycle: mind wandering (MW), awareness of MW, shifting of attention, and sustained attention. Using subjective input from experienced practitioners during meditation, we identified activity in salience network regions during awareness of MW and executive network regions during shifting and sustained attention. Brain regions associated with the default mode were active during $\mathrm{MW}$. In the present study, we reasoned that repeated activation of attentional brain networks over years of practice may induce lasting functional connectivity changes within relevant circuits. To investigate this possibility, we created seeds representing the networks that were active during the four phases of the earlier study, and examined functional connectivity during the resting state in the same participants. Connectivity maps were then contrasted between participants with high vs. low meditation experience. Participants with more meditation experience exhibited increased connectivity within attentional networks, as well as between attentional regions and medial frontal regions. These neural relationships may be involved in the development of cognitive skills, such as maintaining attention and disengaging from distraction, that are often reported with meditation practice. Furthermore, because altered connectivity of brain regions in experienced meditators was observed in a non-meditative (resting) state, this may represent a transference of cognitive abilities "off the cushion" into daily life.

Keywords: functional connectivity, attention, meditation, default mode, networks, fMRI

\section{INTRODUCTION}

Various forms of contemplative practice, including meditation, have recently become the subject of intensive scientific research (Ospina et al., 2007; Chiesa, 2009, 2010; Rubia, 2009; Chiesa and Serretti, 2010; Green and Turner, 2010). From a neuroscience perspective, one of the main research goals is to determine which brain regions mediate the cognitive aspects of various practices. Evidence is now accumulating to suggest that attentional brain regions are involved in many meditative practices (BrefczynskiLewis et al., 2007; Hölzel et al., 2007; Pagnoni and Cekic, 2007; Lutz et al., 2009; Baron Short et al., 2010; Manna et al., 2010; BerkovichOhana et al., 2011; Kilpatrick et al., 2011; Xue et al., 2011; Yu et al., 2011; Hasenkamp et al., 2012; Kozasa et al., 2012). This is perhaps not surprising, given that most meditation practices require some amount of focused attention (FA) and/or executive mental functions (Lutz et al., 2008). However, not all studies agree on which regions are active during meditation, and it is likely that findings are dependent on the particular practice studied and methodology used. Driving this inquiry into how meditation affects the brain is the larger aim of understanding how repeated practice leads to beneficial mental and physical effects in daily life. Studies increasingly suggest that some meditation-related benefits are associated with the amount of practice a person has undertaken
(Brefczynski-Lewis et al., 2007; Pace et al., 2009; Baron Short et al., 2010; Manna et al., 2010). In general, it is reasonable to postulate that as meditation experience accumulates, the repeated engagement of various cognitive functions and associated brain networks induces neuroplastic changes that mediate positive outcomes.

Recently, we developed a cognitive model and fMRI paradigm to examine the neural underpinnings of brief mental states that are involved in FA meditation (Hasenkamp et al., 2012). FA practice is intended to help the practitioner enhance awareness of cognitive states while developing attentional control (Lutz et al., 2008). During FA practice, an individual attempts to maintain focus on a single object (e.g., the breath), and brings attention back to the object whenever the mind wanders (Gunaratana, 2002; Wallace, 2006). In relation to the broader scope of meditation practices, FA meditation is often used as a basic and foundational practice, with the goal of training various aspects of attentional control before undertaking more advanced or specific practices (Lutz et al., 2008). As such, forms of this practice are common to many contemplative traditions, with common objects of attentional focus ranging from somatosensory (e.g., the breath) to a sound or visual stimulus. Our model proposes that during FA meditation, one's subjective experience follows the structure summarized in Figure 1A. When attempting to sustain focus on an 

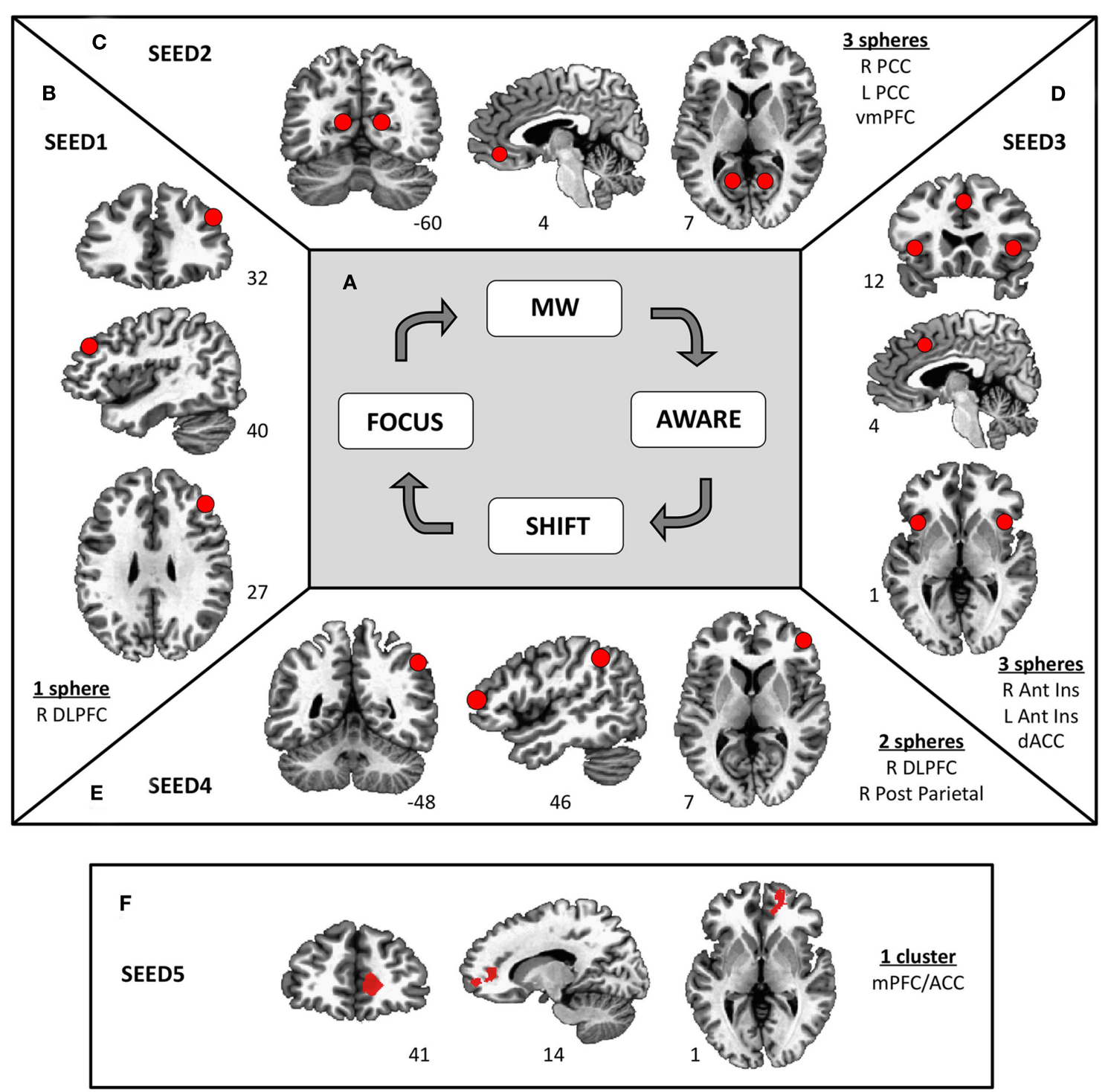

FIGURE 1 | Functional connectivity seeds. (A) A four-stage model of cognitive states experienced during FA meditation. Sustained focus on an object (FOCUS) is inevitably interrupted by mind wandering (MW). At some time during $\mathrm{MW}$, awareness arises that the mind is not on the object (AWARE), followed by disengagement from the current train of thought and shifting of attention (SHIFT) back to the object (FOCUS). Previous results show differential brain activity for these four phases (see Appendix; Hasenkamp et al., 2012). Six millimeter spheres were drawn around the center of the activated clusters of interest to yield functional connectivity seeds. (B) On the activations from the FOCUS phase, an ROI was constructed around the right dIPFC (SEED1). (C) On the activations from the MW phase, ROls were constructed around the ventromedial PFC and bilateral posterior cingulate cortex (SEED2). (D) On the activations from the AWARE phase, ROls were constructed around the dorsal ACC and bilateral anterior insula (SEED3). (E) On the activations from the SHIFT phase, ROIs were constructed around the right $\mathrm{dIPFC}$ and the right posterior parietal lobule (SEED4). (F) A cluster in the medial PFC/ACC was found to be less active during the SHIFT condition as participants' meditation experience increased (see Appendix). To further understand this finding, this cluster is included as SEED5. object, such as the breath (FOCUS), an individual inevitably experiences mind wandering (MW). At some time during $\mathrm{MW}$, the practitioner becomes aware that his/her mind is not on the object (AWARE), at which point he/she disengages from the current train of thought and shifts attention back to the object (SHIFT), where it stays focused again for some period of time (FOCUS). In our previous study (Hasenkamp et al., 2012), we asked experienced meditation practitioners to perform 20 min of breath-based FA meditation while undergoing fMRI scanning, with instructions to press a button whenever they realized their mind had wandered, and then return their focus to the breath. Thus, the button presses in this task provided temporal information on moments of naturally occurring awareness of MW.

The mental processes of MW and attention are increasingly becoming associated with activity in different distributed brain networks. A task-negative, or default mode network (DMN) 
has been associated with task-independent, spontaneous thought processes, also known as MW (Buckner et al., 2008). MW processes are directed away from a primary task and toward personal goals, and cover a broad range of mental functions, including memory, planning, and theory of mind (Smallwood and Schooler, 2006). The DMN consists of hubs in the medial prefrontal cortex (mPFC) and posterior cingulate cortex, and also includes inferior parietal and lateral temporal regions (Buckner et al., 2008). The mPFC has been specifically implicated in self-related cognitive processing (Northoff and Bermpohl, 2004), which is arguably a central feature of much MW experience. Conversely, a task-positive network has been associated with various attentional, present moment, and task-related processes, and is active during rest in an anticorrelated manner with the DMN (Fox et al., 2005; Fransson, 2005). This large task-positive attention network can be subdivided in several ways to yield smaller and more distinct subnetworks. One such division distinguishes the salience and executive networks (Seeley et al., 2007). The salience network is thought to be involved in the immediate, present moment processing, or detection of relevant stimuli (e.g., a state of MW during meditation), and involves the dorsal anterior cingulate cortex (ACC) and bilateral anterior insula (Seeley et al., 2007; Craig, 2009). The executive network, also referred to as the fronto-parietal attention network, consists of dorsolateral prefrontal cortex (dlPFC) and postero-lateral parietal regions, and is involved in controlling attentional resources to deal with immediate or future demands (Seeley et al., 2007; Corbetta et al., 2008).

Using a subject-driven approach to fMRI analysis, we identified patterns of neural activation during each of the four cognitive phases of FA meditation described above. Specifically, activity was detected in brain regions associated with attentional subnetworks during AWARE, SHIFT, and FOCUS phases. During the AWARE phase, robust activations were observed in the salience network. During the SHIFT and FOCUS phases, elements of the executive network were active. We also detected activity during the MW phase in brain regions frequently associated with the DMN, mentalizing, and self-related processing. This overall pattern of network activations is consistent with cyclic alternation between default mode and task-positive networks, in which DMN activity is associated with MW, and attentional subnetworks are associated with awareness, shifting attention, and maintaining attention.

Detailed temporal analyses such as this can be useful in understanding the moment-to-moment cognitive shifts underlying the subjective experience of meditation. However, to understand how cognitive skills gained during practice may be transferred "off the cushion," we must also investigate brain states more reflective of daily life. In the present study, we reasoned that the networks involved in FA meditation (as identified by our previous study) would be most likely to have undergone meditation-related plasticity after repeated practice. Thus, these regions may express differential resting state functional connectivity depending on the amount of meditation experience a person has accumulated. To investigate this possibility, we created seeds to represent the activation patterns seen in each of the four conditions above. We then used these seeds to perform functional connectivity analysis on resting state data from the same participants. Participants were dichotomized into high and low practice groups, and results were compared between groups to evaluate the effect of meditation experience on the functional connectivity of these networks. We hypothesized that participants with more meditation experience would exhibit increased functional connectivity of attentional networks, possibly reflecting plasticity induced by repeated engagement of these networks during contemplative practice.

\section{MATERIALS AND METHODS PARTICIPANTS}

Fourteen healthy right-handed non-smoking meditation practitioners, ages 28-66 (three male), were recruited from local Atlanta meditation communities (see Appendix for information on specific contemplative traditions). All participants signed a consent form approved by the Institutional Review Board at Emory University and the Atlanta Veterans Affairs Research and Development Committee as an indication of informed consent. Participants were assessed for meditation experience to estimate lifetime practice hours and ensure familiarity with breath-focus meditation. Information on estimation of meditation experience, as well as other demographic variables, is provided in Appendix. Exclusion criteria were: less than 1 year of regular meditation practice, fMRI contraindications, current substance dependence, history of sustained loss of consciousness, major neurological or medical illness, left-handedness, pregnancy, or history of major mental illness (as assessed by the Structured Clinical Interview for DSM-IV, Axis-I, Non-Patient version; First et al., 2001).

\section{MRI DATA ACQUISITION AND PRE-PROCESSING}

Images were collected at the Emory Biomedical Imaging Technology Center on a 3-T Siemens Trio scanner using a 12channel head coil and parallel imaging with an iPAT acceleration factor of 2 . Head movement was minimized with foam padding around the head. Functional images were obtained using a T2* weighted gradient-echo pulse sequence $(\mathrm{TR}=1500 \mathrm{~ms}$, $\mathrm{TE}=30 \mathrm{~ms}$, flip angle $=90^{\circ}, \mathrm{FOV}=192 \mathrm{~cm}, 64 \times 64$ matrix, voxel dimensions $=3 \mathrm{~mm} \times 3 \mathrm{~mm} \times 4 \mathrm{~mm}$ ), providing whole brain coverage in 18 slices. High-resolution anatomical T1weighted images were acquired for localization of neural activations $(\mathrm{TR}=2600 \mathrm{~ms}, \mathrm{TE}=3.9 \mathrm{~ms}, \mathrm{TI}=900 \mathrm{~ms}, \mathrm{FOV}=24 \mathrm{~cm}$, $256 \times 256$ matrix, voxel dimensions $=1 \mathrm{~mm} \times 1 \mathrm{~mm} \times 1 \mathrm{~mm}$ ). Resting state scans were collected over $7 \mathrm{~min}$ with eyes closed. During this scan, the participant was asked to relax, and specifically not to engage in meditation. All participants confirmed they were indeed able to refrain from meditating, suggesting that the cognitive experience during this resting state scan is comparable to other resting state studies.

Functional data were preprocessed using standard methods in AFNI (Cox, 1996). Initial pre-processing steps of the functional data included slice time correction and motion correction, in which all volumes were registered spatially to the 20th volume in the functional run. The functional data were next smoothed using an isotropic $8 \mathrm{~mm}$ full-width half-maximum Gaussian kernel. To minimize global drift effects, the signal intensities in each volume were divided by the mean signal value for the run and multiplied by 100 to produce percent signal change from the run mean. All functional datasets were then resampled onto a $2-\mathrm{mm} \times 2$ $\mathrm{mm} \times 2-\mathrm{mm}$ grid and bandpass filtered $(0.01-0.08 \mathrm{~Hz})$ to reduce 
effects of low-frequency drift and high-frequency noise. This final dataset was used for all functional connectivity analyses described below. The anatomical scan was corrected for image intensity non-uniformity, skull-stripped, and then aligned with the functional data. The resulting aligned anatomical dataset was warped to Talairach space using an automated procedure employing the TT_N27 template.

\section{PREVIOUS EXPERIMENT: MEDITATION TASK AND ANALYSIS}

To explain our motivation for constructing the seeds for the current functional connectivity analysis, we first briefly review our previous experiment; for full details on the meditation task and subsequent analyses, see Hasenkamp et al. (2012). Briefly, participants were asked to meditate for $20 \mathrm{~min}$ in the scanner by maintaining FA on the breath (specifically on the sensations of the breath on the nostrils and upper lip), keeping the eyes closed. They were instructed to press a button whenever they realized that their mind had wandered away from the breath, and then return their focus to the breath. MW was construed as noticing when the mind was completely off the breath, being fully absorbed in a train of thought. Participants were all familiar with basic breath-focus meditation, readily understanding and performing the task. Participants practiced the button-pressing task outside the scanner extensively beforehand, so that they were comfortable performing it in the scanner.

Based on the model of cognitive fluctuations in Figure 1A, we constructed a cognitively defined baseline and three 3-s intervals surrounding each button press (see Figure A1 in Appendix). The TR containing the button press, as well as the preceding TR, constituted the AWARE phase, corresponding to awareness of MW (3s total). The two TRs ( $3 \mathrm{~s}$ ) before the AWARE phase were cognitively defined as MW, representing loss of focus, and were treated as the baseline in the general linear model (GLM). The two TRs (3s) following the AWARE phase made up the SHIFT phase, representing the shifting of attention back to the breath. Finally, the two TRs (3s) following the SHIFT phase made up the FOCUS phase, representing maintenance of FA on the breath. A 6-s regressor of no interest after the FOCUS phase was included to model the remainder of the hemodynamic response function (HRF) following the button press. Importantly, all time points not included in one of these phases were censored from the analysis, as they contained data that corresponded to undefined mental states.

A beta value was obtained at each voxel for each condition of interest by fitting a GLM to each participant's percent signal change data. The GLM included: (1) regressors for three conditions of interest (AWARE, SHIFT, and FOCUS) modeled by convolving box car functions of the relevant time frame with a canonical gamma HRF (the fourth condition, MW, was the baseline); (2) a basis set of second order polynomial functions, modeling low-frequency confounds; and (3) the subject's motion parameters, treated as confounds. The betas for the conditions of interest from each participant's regression analysis were warped to Talairach space and entered into a second-level random effects ANOVA with conditions of interest as fixed effects and participants as a random effect.

The main findings from the initial analysis are re-printed as Figure A2 in Appendix, showing AWARE, SHIFT, and FOCUS activations contrasted with the MW baseline, and MW activations defined from the MW > SHIFT contrast. As described earlier, we detected activity in brain regions associated with the salience network activity during the AWARE phase, the executive network in the FOCUS and SHIFT phases, and the DMN during the MW phase.

\section{CONSTRUCTION OF SEEDS}

In the present study, functional connectivity seeds were constructed to represent the main nodes of brain activity in each of the four phases described above. Because our previous findings contained important elements of the salience, executive, and DMNs, we created seeds that reflected these networks as defined by our earlier results ${ }^{1}$. These seeds are summarized in Figure 1. In some cases, multiple spheres from different important regions of the same network were included in its seed. To make the seeds, $6 \mathrm{~mm}$ spheres were drawn in the activated clusters of interest, such that the center of the sphere was a close as possible to the peak voxel, while also ensuring that (1) the full volume of the sphere contained activated voxels and (2) bilateral regions were aligned in coronal and axial planes. For the activations from the FOCUS phase, a sphere was constructed around the right dlPFC (SEED1, one sphere; Figure 1B); for the activations from the MW phase, spheres were constructed around the ventromedial PFC and bilateral posterior cingulate cortex (SEED2, three spheres; Figure 1C); for the activations from the AWARE phase, spheres were constructed around the dorsal ACC and bilateral anterior insula (SEED3, three spheres; Figure 1D); and for the activations from the SHIFT phase, spheres were constructed around the right dlPFC and the right posterior parietal lobule (SEED4, two spheres; Figure 1E).

In addition to the task-based analysis described in Section "MRI Data Acquisition and Pre-processing," we previously performed a correlation analysis to examine the relationship of meditation experience to brain activity in each cognitive phase of the meditation task. In the SHIFT phase, a cluster in the medial PFC/ACC became less active as participants' meditation experience increased (Figure A4A in Appendix). This cluster was investigated further in our previous work by modeling the HRF in this region. These modeling results showed that activity in this region decreased over time during SHIFT in participants with high practice, but persisted in participants with low practice (Figure A4B in Appendix). To further understand this finding, we investigated functional connectivity associated with this region here. This cluster is therefore included as the final seed in this study (SEED5; Figure 1F).

\section{FUNCTIONAL CONNECTIVITY ANALYSIS}

For each participant, the anatomical image was segmented using FSL (Smith et al., 2004) into cerebrospinal fluid (CSF), gray matter, and white matter segments. To control for non-specific signal, time series were extracted from the CSF and white matter segments of each subject's final dataset and included as regressors of no interest in analyses as noted below. Time series were extracted

\footnotetext{
${ }^{1}$ It should be noted that our results are in close agreement with the literature about the regions that comprise the networks in question (Fox et al., 2005; Fransson, 2005; Seeley et al., 2007; Buckner et al., 2008).
} 
from each of the five seeds (described above, and see Figure 1) using each participant's final resting state data, and artifact was removed by regressing six standard motion parameters, as well as CSF and white matter signal. For each seed, functional connectivity was calculated by fitting a GLM to each participant's resting state dataset. The GLM included: (1) the cleaned seed time series as the regressor of interest; (2) a basis set of second order polynomial functions, modeling low-frequency confounds; (3) the participant's motion parameters, treated as confounds; and (4) the two time series extracted from the participant's CSF and white matter segments, treated as confounds.

Participants were dichotomized into high and low practice groups, as practice time was distributed bimodally in this sample (Figure A3 in Appendix), with five "high practice" participants having greater than $2000 \mathrm{~h}$ of experience and nine "low practice" participants having less than $1200 \mathrm{~h}$ ( $p<0.001$, Table A1 in Appendix). Overall, the groups were well matched on age, gender, and personality characteristics (Table A1 in Appendix). Group level analysis for each seed was performed using $t$-tests on the functional connectivity maps of each group. The voxel-wise significance level was $p<0.005$ with a spatial extent threshold of 20 functional voxels.

\section{RESULTS}

Table 1 reports clusters that differed in functional connectivity between high and low practice groups for the seeds defined in Figure 1. The results for each seed are addressed in turn.

\section{SEED1}

SEED1 was derived from activations during sustained attention, and consisted of the right dlPFC. Functional connectivity to SEED1 was stronger in high practice participants for three clusters in the right insula, as well as for a region in the left dlPFC and in the mid-cingulate gyrus (Figure 2A). Functional connectivity was reduced in high practice participants between the right dlPFC and several visual regions (cuneus and middle occipital gyrus), as well as for a cluster in the right dlPFC, slightly ventral to the seed itself.

\section{SEED2}

SEED 2 was derived from activations during MW (MW > SHIFT), and was comprised of three spheres representing the major hubs of the DMN (ventromedial PFC and bilateral posterior cingulate cortex). Using this seed, only two regions were identified as having differential functional connectivity between high and low practice groups. Connectivity to the left ventromedial orbitofrontal gyrus was stronger in the high practice group, whereas connectivity to the left ACC was reduced in the high practice group (Figure 2B).

\section{SEED3}

SEED3 was derived from activations seen at the moment participants became aware of MW, and contained three spheres representing the salience network (dorsal ACC and bilateral anterior insula). In the high practice group, SEED3 was more functionally connected to the left cingulate gyrus and to the left inferior parietal lobule (Figure 2C). Conversely, connectivity in this group was reduced from this seed to the right middle occipital gyrus, cerebellum, and left postcentral gyrus.

\section{SEED4}

SEED4 was derived from activations during the disengagement and re-orienting of attention back to the breath, and contained two spheres representing the executive network (right dlPFC and right inferior parietal lobule). In the high practice group, functional connectivity from this seed was increased to the left dlPFC, and was reduced to the right middle occipital gyrus and cerebellum (Figure 2D).

\section{SEED5}

SEED5 was derived from a mPFC/ACC cluster identified as being less active during the SHIFT phase for high practice participants than for low practice participants. This region is also often included in the DMN (Buckner et al., 2008). For SEED5, high practice participants exhibited increased connectivity to many regions, including bilateral interior parietal lobules, left precentral gyrus, left interior frontal gyrus, left orbitofrontal cortex, and right dlPFC (Figure 2E). Reduced connectivity from SEED5 for the high practice group was seen in left posterior cingulate cortex and left lingual gyrus.

\section{DISCUSSION}

This study focused on functional connectivity of brain regions and networks that were implicated in our earlier study of FA meditation (Hasenkamp et al., 2012). Using resting state data and comparing participants with higher or lower lifetime estimated hours of practice, we investigated connectivity that may be related to meditation experience. Below, we reflect on general patterns that can be seen considering multiple networks. Specifically, we consider the relationship of meditation experience to functional connectivity in attention networks and in the DMN.

\section{FUNCTIONAL CONNECTIVITY WITHIN ATTENTION NETWORKS}

Overall, the present analysis revealed many instances of participants with greater meditation experience having increased functional connectivity among attentional regions. As the practice of FA meditation is primarily a form of attention training, this suggests that brain regions mediating the cognitive functions required for this practice may be undergoing experience-dependent plasticity that is evident in the resting state. For example, the right dIPFC (SEED1) was active during the FOCUS condition of FA meditation in our previous analysis. This region is an important hub of the fronto-parietal executive network, and is also known to be involved in working memory (Curtis and D'Esposito, 2003; Seeley et al., 2007; Corbetta et al., 2008). Given that the cognitive state experienced during the FOCUS phase (i.e., sustained FA) likely occupies a large percentage of time spent in meditation, it is reasonable to hypothesize that the circuitry involved in mediating this function would be most likely to undergo neuroplastic changes as a result of repeated practice. Indeed, functional connectivity of the seed derived from this cognitive phase (SEED1) exhibited many differences between high and low practice groups, suggesting that this region may be one of the most influenced by meditation experience.

A notable result for SEED1 was increased connectivity in high practice participants to three regions in the right insula (Figure 2A). The insula has been implicated in a vast array 
Table 1 | Functional connectivity between groups.

\begin{tabular}{|c|c|c|c|c|c|c|}
\hline & \multirow{2}{*}{$\frac{\text { Brodmann }}{\text { Area }}$} & \multirow[t]{2}{*}{ Voxels } & \multicolumn{3}{|c|}{ Peak } & \multirow{2}{*}{$\frac{\text { Mean }}{t \text {-Value }}$} \\
\hline & & & $x$ & $Y$ & $z$ & \\
\hline \multicolumn{7}{|l|}{ SEED1 (FOCUS: R DLPFC) ${ }^{a}$} \\
\hline \multicolumn{7}{|l|}{ High practice $>$ low practice } \\
\hline Cingulate gyrus & 23 & 249 & -3 & -27 & 30 & 4.31 \\
\hline$L$ mid frontal gyrus & 9 & 106 & -31 & 11 & 30 & 4.50 \\
\hline $\mathrm{R}$ post insula/sup temporal gyrus & 13,21 & 59 & 39 & -5 & -8 & 3.98 \\
\hline R Ant mid insula & 13 & 50 & 31 & 9 & -6 & 4.09 \\
\hline R Ant insula/frontal operculum & 13,47 & 45 & 39 & 25 & -2 & 3.84 \\
\hline \multicolumn{7}{|l|}{ Low practice $>$ high practice } \\
\hline R mid occipital gyrus & 19 & 334 & 39 & -73 & 16 & -3.83 \\
\hline R mid frontal gyrus & 9 & 45 & 49 & 29 & 30 & -4.24 \\
\hline L mid occipital gyrus & 19 & 37 & -39 & -83 & 14 & -3.78 \\
\hline R cuneus & 18 & 30 & 21 & -95 & 12 & -3.72 \\
\hline \multicolumn{7}{|c|}{ SEED2 (MW: DEFAULT MODE NETWORK) } \\
\hline \multicolumn{7}{|c|}{ High practice $>$ low practice } \\
\hline L vmPFC/OFC & 11,25 & 22 & -13 & 31 & -14 & 3.62 \\
\hline \multicolumn{7}{|l|}{ Low practice $>$ high practice } \\
\hline $\mathrm{L}$ ant cingulate & 24 & 65 & -3 & 33 & 10 & -3.92 \\
\hline \multicolumn{7}{|c|}{ SEED3 (AWARE: SALIENCE NETWORK) } \\
\hline \multicolumn{7}{|c|}{ High practice $>$ low practice } \\
\hline $\mathrm{L}$ cingulate gyrus & 23,31 & 63 & 1 & -21 & 26 & 3.94 \\
\hline L Inf parietal lobule & 40 & 20 & -47 & -41 & 48 & 3.90 \\
\hline \multicolumn{7}{|l|}{ Low practice $>$ high practice } \\
\hline Cerebellar declive & - & 282 & -3 & -67 & -18 & -3.90 \\
\hline R mid occipital gyrus & 19 & 79 & 43 & -81 & 8 & -4.05 \\
\hline L postcentral gyrus/mid insula & 13 & 58 & -47 & -11 & 18 & -3.77 \\
\hline \multicolumn{7}{|c|}{ SEED4 (SHIFT: EXECUTIVE NETWORK) } \\
\hline \multicolumn{7}{|c|}{ High practice $>$ low practice } \\
\hline $\mathrm{L}$ mid frontal gyrus & 9 & 121 & -31 & 13 & 24 & 4.26 \\
\hline \multicolumn{7}{|l|}{ Low practice $>$ high practice } \\
\hline R mid occipital gyrus & 18 & 34 & 25 & -93 & 6 & -3.94 \\
\hline Cerebellar declive & - & 27 & 1 & -71 & -10 & -3.57 \\
\hline \multicolumn{7}{|l|}{ SEED5 (MPFC/ACC) ${ }^{\mathrm{b}}$} \\
\hline \multicolumn{7}{|l|}{ High practice $>$ low practice } \\
\hline R Inf parietal lobule & 40 & 137 & 43 & -49 & 54 & 3.65 \\
\hline L precentral gyrus & 4 & 61 & -31 & -17 & 34 & 3.83 \\
\hline $\mathrm{R}$ Inf parietal lobule & 40 & 32 & 45 & -33 & 58 & 4.09 \\
\hline L Inf frontal gyrus/precentral & 44 & 30 & -59 & 7 & 12 & 4.39 \\
\hline L vmPFC/OFC & 11,25 & 23 & -9 & 29 & -14 & 3.96 \\
\hline $\mathrm{R}$ dIPFC & 8 & 22 & -47 & -51 & 50 & 3.79 \\
\hline L Inf parietal lobule & 40 & 22 & 43 & 15 & 42 & 3.71 \\
\hline \multicolumn{7}{|l|}{ Low practice $>$ high practice } \\
\hline L lingual gyrus & 19 & 96 & -25 & -69 & -2 & -3.97 \\
\hline $\mathrm{L}$ post cingulate & 18 & 48 & -23 & -55 & 8 & -3.74 \\
\hline
\end{tabular}

Clusters that were significantly different between high and low practice groups $(p<0.005,20$ functional voxels; voxel size $2 \mathrm{~mm} \times 2 \mathrm{~mm} \times 2 \mathrm{~mm})$. ${ }^{\mathrm{a}}$ The region in SEED1, the right dorsolateral PFC, is a major component of the executive network. "The region in SEED5 does not represent a "network," but was derived from our previous analysis, which showed activity in this region was correlated with practice time in these subjects. See Materials and Methods for more explanation. $R$, right; $L$, left. If $R$ or $L$ not listed, regions are bilateral. Ant, anterior; dIPFC, dorsolateral prefrontal cortex; Inf, inferior; Mid, middle; OFC, orbitofrontal cortex; Post, posterior; vMPFC, ventromedial prefrontal cortex.

of tasks, including present moment awareness and sensation of internal states (Craig, 2002, 2009). Increased coherence of signal between right dlPFC and right insula at rest suggests that individuals with more meditation experience may have an enhanced 

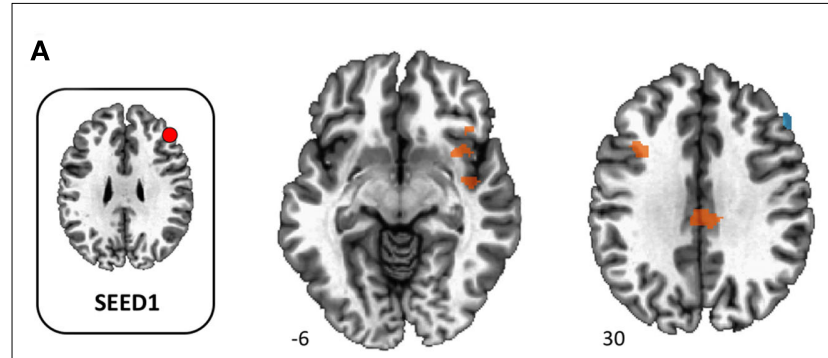

B
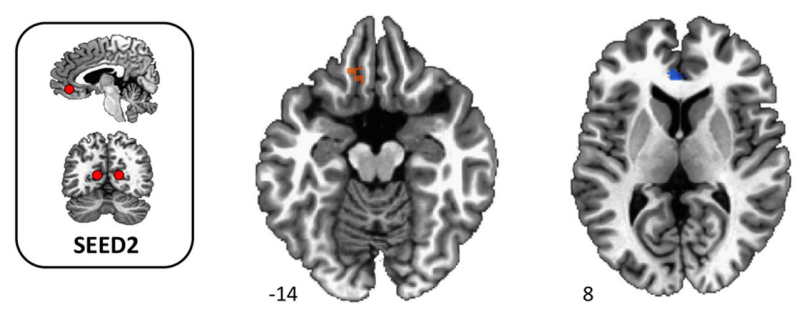

C
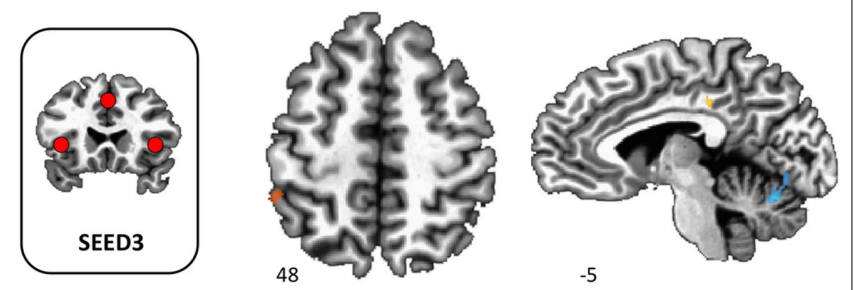

D
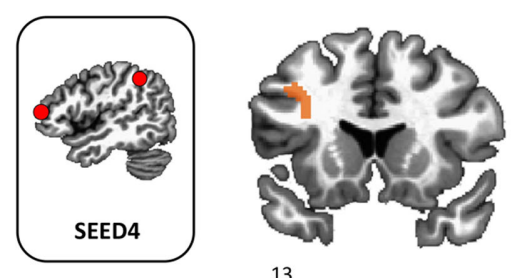

13

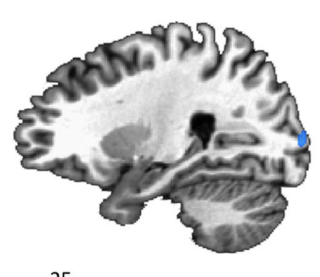

25

E
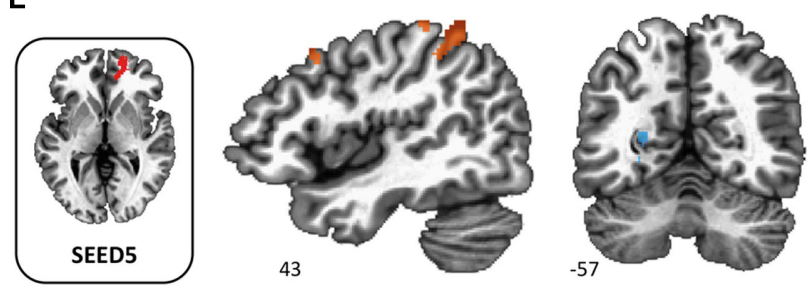

increased connectivity with more meditation experience decreased connectivity with more meditation experience

FIGURE 2 | Functional connectivity differences between high and low practice participants. (A) Increased functional connectivity in high practice participants between SEED1 and right insula, left dIPFC, and mid-cingulate. (B) Increased functional connectivity in high practice participants between SEED2 and left orbitofrontal cortex; decreased functional connectivity in

(Continued)

\section{FIGURE 2 | Continued}

high practice participants between SEED1 and ACC. (C) Increased functional connectivity in high practice participants between SEED3 and left inferior parietal lobule and left middle cingulate gyrus; decreased functional connectivity in high practice participants between SEED3 and cerebellar declive. (D) Increased functional connectivity in high practice participants between SEED4 and left middle frontal cortex; decreased functional connectivity in high practice participants between SEED4 and right middle occipital gyrus. (E) Increased functional connectivity in high practice participants between SEED5 and left inferior parietal lobule; decreased functional connectivity in high practice participants between SEED5 and left posterior cingulate cortex.

awareness of present moment experience and more access to internal bodily states when employing executive processes in daily life. Interestingly, the finding of increased PFC-insula connectivity has previously been associated with meditation experience (Farb et al., 2007). In that study, participants who had undergone 8 weeks of training in mindfulness showed greater right insula activation than novices during an experiential focus task, and had stronger functional connectivity than novices between right insula and dlPFC. Our similar connectivity finding in a different population of practitioners, using seeds identified through different methodology, suggests that these changes may be consistently associated with meditation.

From a network perspective, right anterior insula has also been implicated as a region that is central for switching between the DMN and the executive network (Sridharan et al., 2008). The ability to switch between states of MW (DMN-related), and states of focus (executive network-related) is a central skill that is trained in FA meditation (Lutz et al., 2008); indeed, this is one of the primary objectives of the practice. Increased connectivity at rest between the right insula and elements of the executive network in experienced meditators may allow for a more efficient switching between these networks and their associated mental states in daily life.

Aside from group differences in insula connectivity, increased connectivity from right dlPFC (SEED1) to left dlPFC was seen in those with greater experience, perhaps suggesting an increased bilaterality of executive function in these participants (Figure 2A). Further, the seed representing the salience network (SEED3) showed increased meditation-related functional connectivity to left inferior parietal lobule, an element of the executive network (Figure 2C). Speculating on implications of this general trend, it is possible that increased functional connectivity between attentional systems in the brains of experienced meditators allows for enhanced attentional processing, which has been shown behaviorally in a number of studies (Jha et al., 2007; Lutz et al., 2009; Zeidan et al., 2010).

\section{FUNCTIONAL CONNECTIVITY WITHIN THE DEFAULT MODE NETWORK}

Additional results show that meditation experience is associated with connectivity differences between the anterior and posterior elements of the DMN. First, the seed representing the default mode regions active during self-identified MW (SEED2) showed decreased connectivity to the ACC in those with greater meditation experience (Figure 2B). These findings are also reflected in the 
results of the SEED5 analysis, where a similar ACC region (SEED5) exhibited reduced connectivity to the left posterior cingulate cortex (part of SEED2) in the high practice group (Figure 2E). This kind of decoupling between anterior and posterior elements of the DMN has been shown previously with regard to meditation training. Specifically, when Kilpatrick et al. (2011) assessed functional connectivity in MBSR participants compared to controls, they found that, in MBSR participants, activity in a very similar ACC region showed increased negative correlation with the posterior cingulate regions in our SEED2.

Furthermore, two analyses found increased meditationrelated functional connectivity between DMN regions and left orbitofrontal cortex/ventromedial PFC (Table 1; Figure 2B), for both SEED2 and SEED5 (again, both seeds represent DMN regions) ${ }^{2}$.Consistent with this finding, Jang et al. (2011) recently studied meditators who practiced "focusing attention on their bodily sensations and emotions, and heightening awareness of the movement of energy within the body," and found that the DMN showed increased connectivity to the orbitofrontal cortex/ventromedial PFC in meditators compared to controls. This area of the brain is highly interconnected with limbic regions, and has been implicated in processing viscero-motor states, as well as in computing expected value, reward outcome, and subjectively experienced pleasure (Ongur and Price, 2000; Grabenhorst and Rolls, 2011). Increased connectivity between medial orbitofronal cortex and DMN regions in more experienced meditators might suggest that their default circuitry has greater access to information about internal states, and may also enable more efficient reward/value processing. Perhaps when experienced meditators are MW, increased access to visceral signal increases awareness of MW and facilitates switching back to FA. As described earlier, the salience network is implicated in switching between default and executive networks; indeed, medial areas of the orbitofrontal cortex are highly interconnected to the salience network hubs, the ACC, and insula (Ongur and Price, 2000; Grabenhorst and Rolls, 2011). Thus, increased coherence of the DMN with the medial orbitofrontal cortex could allow for more efficient switching from the DMN to executive network via the salience network.

\section{FUNCTIONAL CONNECTIVITY BETWEEN ATTENTION AND DEFAULT MODE NETWORKS}

The cluster comprising SEED5 was identified in a previous correlation analysis, where it was found to have reduced activity during the SHIFT phase of the FA meditation cycle in high practice participants, exhibiting a faster return to baseline (Figure A4 in Appendix; Hasenkamp et al., 2012). We interpreted that finding as showing that individuals with greater meditation experience may more efficiently disengage from ongoing MW or self-related thinking that this frontal region mediated. Results from the present analysis suggest one mechanism that could allow for this. In high practice participants, SEED5 showed increased functional connectivity to bilateral regions of the inferior parietal lobule (Table 1; Figure 2E). These parietal regions are part of the executive network, and have

${ }^{2}$ While this region can be subject to signal loss in fMRI experiments, a comparison of signal-to-noise ratio maps between high and low practice participants showed no difference in signal between groups in this region. been specifically implicated in attentional disengagement (Posner et al., 1984). With increased coherence between these regions, experienced meditators may have improved capacity for disengagement of thought content mediated by the medial PFC region. Stronger resting state functional connectivity between mPFC and right inferior partial lobule was also recently found in experienced meditators as compared to beginners (Taylor et al., in press), again suggesting that this may be a consistent effect of meditation.

\section{OTHER REGIONS}

In addition to the findings reviewed so far, several others suggest a general trend toward lower connectivity between attention and visual networks in participants with more meditation experience (see Table 1: SEED1, SEED3, SEED4). Consistent with these findings, another recent study found decreased functional connectivity between salience regions and visual/occipital regions in MBSR participants compared to controls (Kilpatrick et al., 2011). Together, both sets of results suggest that meditators have may more efficient attentional allocation, with decreased attentional resources being devoted to visual processing during irrelevant situations (e.g., eyes closed).

\section{LIMITATIONS AND FUTURE DIRECTIONS}

Given the small sample size examined here, it is important to remember that these findings must be verified in a larger population, and it is possible that the group differences identified here could be due to factors other than meditation experience. It is encouraging, however, that some of the results we obtained have also been reported in other studies (as reviewed above), suggesting consistent neural effects of meditation. It should also be noted that estimates of lifetime meditation experience in this population are necessarily imprecise, with an individual's experience likely including other practices in addition to strict FA meditation (see Appendix). Importantly, the present work is also subject to potential confounds inherent in any cross-sectional design. For example, it cannot be determined whether effects associated with meditation experience are in fact caused by the practice. To the contrary, these group differences may have existed before meditation was ever undertaken, perhaps predisposing certain individuals to practice more often, more effectively, etc. In the future, longitudinal studies must be employed to establish the causality underlying these brain differences. Further, studies could also correlate clinical outcomes with neural changes in the same population, to examine whether and how meditation-related neuroplasticity is beneficial. Finally, it should be noted that the cognitive cycle involved in FA meditation, switching from focus to distraction and back again, is likely similar to the process involved in a wide range of attention tasks. Thus, it would strengthen the current findings to investigate the effect of meditation on the activity and connectivity of these brain networks in the context of other active tasks.

\section{CONCLUSION}

As the search to understand the biological underpinnings of meditation progresses, one of the important unanswered questions is: how does contemplative training transfer "off the cushion" and bring benefit to daily life? It is generally thought that repeated engagement of relevant brain networks over time induces neuroplastic changes that mediate positive cognitive, emotional, and 
behavioral outcomes. The present results add to growing evidence that the amount of time an individual spends practicing meditation is associated with activity and connectivity changes in the brain, particularly in attentional regions. This study used seeds derived from distinct cognitive phases identified during FA meditation, and examined functional connectivity during the resting state in the same participants. Association with practice time in these findings may therefore represent one mechanism by which the brain networks trained during meditation can be re-wired to have lasting changes that extend into non-meditative mental experiences (e.g., a resting state). Future studies should extend this method of investigation using a longitudinal design, and correlate neural effects with clinical/wellness outcomes. In addition, it would be interesting to investigate the effects of other

\section{REFERENCES}

Baron Short, E., Kose, S., Mu, Q., Borckardt, J., Newberg, A., George, M. S., and Kozel, F. A. (2010). Regional brain activation during meditation shows time and practice effects: an exploratory FMRI study. Evid. Based Complement Alternat. Med. 7, 121-127.

Berkovich-Ohana, A., Glicksohn, J., and Goldstein, A. (2011). Mindfulnessinduced changes in gamma band activity-implications for the default mode network, self-reference and attention. Clin. Neurophysiol. [Epub ahead of print].

Brefczynski-Lewis, J. A., Lutz, A., Schaefer, H. S., Levinson, D. B., and Davidson, R. J. (2007). Neural correlates of attentional expertise in long-term meditation practitioners. Proc. Natl. Acad. Sci. U.S.A. 104, 11483-11488.

Buckner, R. L., Andrews-Hanna, J. R., and Schacter, D. L. (2008). The brain's default network: anatomy, function, and relevance to disease. Ann. N. Y. Acad. Sci. 1124, 1-38.

Chiesa, A. (2009). Zen meditation: an integration of current evidence. J. Altern. Complement. Med. 15, 585-592.

Chiesa, A. (2010). Vipassana meditation: systematic review of current evidence. J. Altern. Complement. Med. 16, 37-46.

Chiesa, A., and Serretti, A. (2010). A systematic review of neurobiological and clinical features of mindfulness meditations. Psychol. Med. 40, 1239-1252.

Corbetta, M., Patel, G., and Shulman, G. L. (2008). The reorienting system of the human brain: from environment to theory of mind. Neuron 58 , 306-324.

Cox, R. W. (1996). AFNI: software for analysis and visualization of functional magnetic resonance neuroimages. Comput. Biomed. Res. 29, 162-173.
Craig, A. D. (2002). How do you feel? Interoception: the sense of the physiological condition of the body. Nat. Rev. Neurosci. 3, 655-666.

Craig, A. D. B. (2009). How do you feelnow? The anterior insula and human awareness. Nat. Rev. Neurosci. 10, 59-70.

Curtis, C. E., and D'Esposito, M. (2003). Persistent activity in the prefrontal cortex during working memory. Trends Cogn. Sci. (Regul. Ed.) 7, 415-423.

Farb, N. A. S., Segal, Z. V., Mayberg, H., Bean, J., McKeon, D., Fatima, Z., and Anderson, A. K. (2007). Attending to the present: mindfulness meditation reveals distinct neural modes of selfreference. Soc. Cogn. Affect. Neurosci. 2, 313-322.

First, M., Spitzer, R. L., Gibbon, M., and Williams, J. (2001). Structured Clinical Interview for DSM-IV-TR Axis I Disorders. New York: Biometrics Research Department, New York State Psychiatric Institute.

Fox, M. D., Snyder, A. Z., Vincent, J. L., Corbetta, M., Van Essen, D. C., and Raichle, M. E. (2005). The human brain is intrinsically organized into dynamic, anticorrelated functional networks. Proc. Natl. Acad. Sci. U.S.A. 102, 9673-9678.

Fransson, P. (2005). Spontaneous lowfrequency BOLD signal fluctuations: an fMRI investigation of the restingstate default mode of brain function hypothesis. Hum. Brain Mapp. 26, 15-29.

Grabenhorst, F., and Rolls, E. T. (2011). Value, pleasure and choice in the ventral prefrontal cortex. Trends Cogn. Sci. (Regul. Ed.) 15, 56-67.

Green, R., and Turner, G. (2010). Growing evidence for the influence of meditation on brain and behaviour. Neuropsychol. Rehabil. 20, 306-311.

Gunaratana, B. (2002). Mindfulness in Plain English. Somerville, MA: Wisdom Publications.

forms of attentional training on these networks. Understanding the neural changes associated with meditation will help clarify the "mechanism of action" of these practices, and allow for more judicious application of such techniques in clinical and wellness settings.

\section{ACKNOWLEDGMENTS}

We extend our warm gratitude to Alexandre Franco, Christine Wilson-Mendenhall, and Giuseppe Pagnoni for their helpful input on the analysis for this study. This work was supported by a Francisco J. Varela Award from the Mind and Life Institute. Additional support came from the Emory Collaborative for Contemplative Studies, the Emory Neuroscience Initiative, and the Atlanta Veterans Affairs Medical Center.

Hasenkamp, W., Wilson-Mendenhall, C. D., Duncan, E., and Barsalou, L. W. (2012). Mind wandering and attention during focused meditation: a fine-grained temporal analysis of fluctuating cognitive states. Neuroimage 59, 750-760.

Hölzel, B. K., Ott, U., Hempel, H., Hackl, A., Wolf, K., Stark, R., and Vaitl D. (2007). Differential engagement of anterior cingulate and adjacent medial frontal cortex in adept meditators and non-meditators. Neurosci. Lett. 421, 16-21.

Jang, J. H., Jung, W. H., Kang, D. H., Byun, M. S., Kwon, S. J., Choi, C. H., and Kwon, J. S. (2011). Increased default mode network connectivity associated with meditation. $\mathrm{Neu}$ rosci. Lett. 487, 358-362.

Jha, A. P., Krompinger, J., and Baime, M. J. (2007). Mindfulness training modifies subsystems of attention. Cogn. Affect. Behav. Neurosci. 7, 109-119.

Kilpatrick, L. A., Suyenobu, B. Y. Smith, S. R., Bueller, J. A., Goodman, T., Creswell, J. D., Tillisch, K., Mayer, E. A., and Naliboff, B. D. (2011). Impact of mindfulnessbased stress reduction training on intrinsic brain connectivity. Neuroimage 56, 290-298.

Kozasa, E. H., Sato, J. R., Lacerda, S. S. Barreiros, M. A., Radvany, J., Russell, T. A., Sanches, L. G., Mello, L. E., and Amaro, E. Jr. (2012). Meditation training increases brain efficiency in an attention task. Neuroimage 59, 745-749.

Lutz, A., Slagter, H. A., Dunne, J. D., and Davidson, R. J. (2008). Attention regulation and monitoring in meditation. Trends Cogn. Sci. (Regul. Ed.) 12, 163-169.

Lutz, A., Slagter, H. A., Rawlings, N. B., Francis, A. D., Greischar, L. L., and Davidson, R. J. (2009). Mental training enhances attentional stability: neural and behavioral evidence. J. Neurosci. 29, 13418-13427.

Manna, A., Raffone, A., Perrucci, M., Nardo, D., Ferretti, A., Tartaro, A., Londei, A., Del Gratta, C., Belardinelli, M. O., and Romani, G. L. (2010). Neural correlates of focused attention and cognitive monitoring in meditation. Brain Res. Bull. 82, 46-56.

Northoff, G., and Bermpohl, F. (2004). Cortical midline structures and the self. Trends Cogn. Sci. (Regul. Ed.) 8, 102-107.

Ongur, D., and Price, J. L. (2000). The organization of networks within the orbital and medial prefrontal cortex of rats, monkeys and humans. Cereb. Cortex 10, 206-219.

Ospina, M., Bond, K., Karkhaneh, M., Tjosvold, L., Vanderneer, B., Liang, Y., Bialy, L., Hooton, N., Buscemi, N., Dryden, D. M., and Klassen, T. P. (2007). Meditation practices for health: state of the research. Evid. Rep. Technol. Assess. (Full Rep). 155, 1-263

Pace, T. W., Negi, L. T., Adame, D. D., Cole, S. P., Sivilli, T. I., Brown, T. D., Issa, M. J., and Raison, C. L. (2009). Effect of compassion meditation on neuroendocrine, innate immune and behavioral responses to psychosocial stress. Psychoneuroendocrinology 34, 87-98.

Pagnoni, G., and Cekic, M. (2007). Age effects on gray matter volume and attentional performance in Zen meditation. Neurobiol. Aging 28, 1623-1627.

Posner, M. I., Walker, J. A., Friedrich, F. J., and Rafal, R. D. (1984). Effects of parietal injury on covert orienting of attention. J. Neurosci. 4, 1863-1874.

Rubia, K. (2009). The neurobiology of meditation and its clinical effectiveness in psychiatric disorders. Biol. Psychol. 82, 1-11. 
Seeley, W. W., Menon, V., Schatzberg, A. F., Keller, J., Glover, G. H., Kenna, H., Reiss, A. L., and Greicius, M. D. (2007). Dissociable intrinsic connectivity networks for salience processing and executive control. J. Neurosci. 27, 2349-2356.

Smallwood, J., and Schooler, J. W. (2006). The restless mind. Psychol. Bull. 132, 946-958.

Smith, S. M., Jenkinson, M., Woolrich, M. W., Beckmann, C. F., Behrens, T. E., Johansen-Berg, H., Bannister, P. R., De Luca, M., Drobnjak, I., Flitney, D. E., Niazy, R. K., Saunders, J., Vickers, J., Zhang, Y., De Stefano, N., Brady, J. M., and Matthews, P. M. (2004). Advances in functional and structural MR image analysis and implementation as FSL. Neuroimage 23(Suppl. 1), S208-S219.
Sridharan, D., Levitin, D. J., and Menon, V. (2008). A critical role for the right fronto-insular cortex in switching between central-executive and default-mode networks. Proc. Natl. Acad. Sci. U.S.A. 105, 12569-12574.

Taylor, V., Daneault, V, Grant, J. A, Scavone, G., Breton, E., Roffe-Vidal, S., Courtemanche, J., Lavarenne, A. S., Marrelec, G., Benali, H., and Beauregard, M. (in press). Impact of mindfulness training on the default mode network during a restful state: a functional connectivity study. Soc. Cogn. Affect. Neurosci. [Manuscript ID: SCAN-11-106].

Wallace, B. A. (2006). The Attention Revolution: Unlocking the Power of the Focused Mind. Somerville: Wisdom Publications.

Xue, S., Tang, Y. Y., and Posner, M. I. (2011). Short-term meditation increases network efficiency of the anterior cingulate cortex. Neuroreport 22, 570-574.

Yu, X., Fumoto, M., Nakatani, Y., Sekiyama, T., Kikuchi, H., Seki, Y., Sato-Suzuki, I., and Arita, H. (2011). Activation of the anterior prefrontal cortex and serotonergic system is associated with improvements in mood and EEG changes induced by Zen meditation practice in novices. Int. J. Psychophysiol. 80 103-111.

Zeidan, F., Johnson, S. K., Diamond, B. J., David, Z., and Goolkasian, P. (2010). Mindfulness meditation improves cognition: evidence of brief mental training. Conscious. Cogn. 19, 597-605.

Conflict of Interest Statement: The authors declare that the research was conducted in the absence of any commercial or financial relationships that could be construed as a potential conflict of interest.

Received: 26 October 2011; accepted: 15 February 2012; published online: 01 March 2012.

Citation: Hasenkamp $W$ and Barsalou LW (2012) Effects of meditation experience on functional connectivity of distributed brain networks. Front. Hum. Neurosci. 6:38. doi 10.3389/fnhum.2012.00038

Copyright () 2012 Hasenkamp and Barsalou. This is an open-access article distributed under the terms of the Creative Commons Attribution Non Commercial License, which permits noncommercial use, distribution, and reproduction in other forums, provided the original authors and source are credited. 


\section{APPENDIX \\ MATERIALS AND METHODS \\ Participants' meditation background}

Participants in this study had primary meditation experience in several Buddhist traditions (Shamatha/breath-focus, Vipassana/insight, and other Tibetan styles such as compassion and tong-len). Several participants had experience in multiple traditions, as is common with Western lay practitioners. Of the 14 total subjects, six primarily practiced Shamatha, five practiced other Tibetan styles (compassion, tong-len), and three practiced Vipassana. Importantly, all of these styles are built on or incorporate breath-focus meditation. Thus, all participants were very familiar with the cognitive experiences of focused attention and mind wandering, and the shifts between them, that occur during breath-focus meditation.

\section{Estimation of lifetime meditation experience}

Lifetime meditation experience was estimated in the following manner. Participants were asked to report their current meditation practice using three variables: days/week $(A)$, minutes/day $(B)$, and years at this frequency $(C)$. Values were assigned to these values as described below. Participants were also asked if there was another time that they had practiced a different style or at a different frequency. If so, they repeated these three variables for the previous practice to yield the values $D, E$, and $F$. Finally, they were asked to list any meditation retreats they had attended, and for each, the number of days and hours/day of practice. The number of days was multiplied by the hours/day and summed over retreats to yield the value $R$. Total lifetime hours was then calculated as follows:

Days/week (assign value $A$, and $D$ if present)

- daily or almost daily $=6$

- $2-4 \times /$ week $=3$

- $\sim 1 \times /$ week $=1$

- $<1 \times /$ week $=0.5$

Minutes/day (assign value $B$, and $E$ if present)

- $5-15 \min =0.12$

- $15-30 \mathrm{~min}=0.33$

- $>30 \mathrm{~min}=0.67$

Years at this frequency (assign value $C$, and $F$ if present) Retreats (assign value $R$ )

- days $\times$ hours/day, summed over retreats

The formula for estimated lifetime hours was:

hours $=[A \times 52 \times B \times C]+[D \times 52 \times \mathrm{E} \times F]+R$

with $D, E$, and $F$ included only if there are values.

Certainly, this method will yield only a rough estimate of actual practice accrued over the lifetime, but we feel it is important to attempt to systematize this calculation in some way, as opposed to asking participants to simply estimate how many hours they have practiced in their lives.

\section{Other demographic variables}

In addition to meditation experience, we also assessed several personality-related variables in these participants. The measures we used were: Five Factor Mindfulness Questionnaire (FFMQ; Baer et al., 2008) to measure overall mindfulness, Response Styles Questionnaire (RSQ; Nolen-Hoeksema and Morrow, 1991) to assess rumination, Calgary symptoms of stress inventory (cSOSI; Carlson and Thomas, 2007) to assess emotional and physical symptoms of stress, and positive and negative affect scale (PANAS; Watson and Clark, 1999) to measure general affect. For each of these scales, in addition to age and practice time, $t$-tests were employed to determine group differences. A Chi-square analysis was used to assess group differences in male:female ratio. Alpha was set at $p=0.05$ for all analyses.

\section{RESULTS}

Group differences on meditation experience and other demographic variables are summarized in Table A1. The high and low practice groups were significantly different on practice time (see Figure A3). No statistical differences were seen between groups on any other measure except Calgary Symptoms of Stress, which showed fewer stress symptoms in the high practice group. This is perhaps not surprising, given strong evidence for the benefits of meditation for stress reduction (e.g., Chiesa and Serretti, 2009). Overall, the general lack of group differences on a range of personality scales supports the suggestion that functional connectivity differences may be due to meditation experience rather than spurious differences between groups.

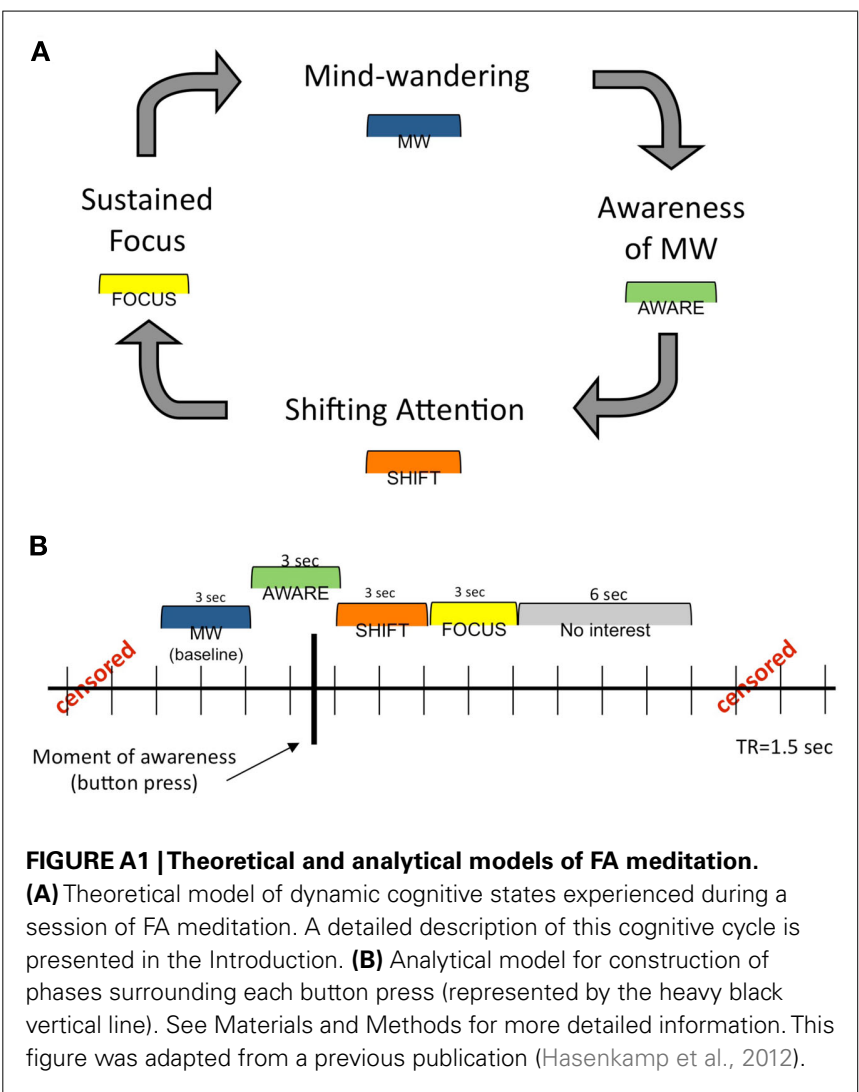




\section{A}
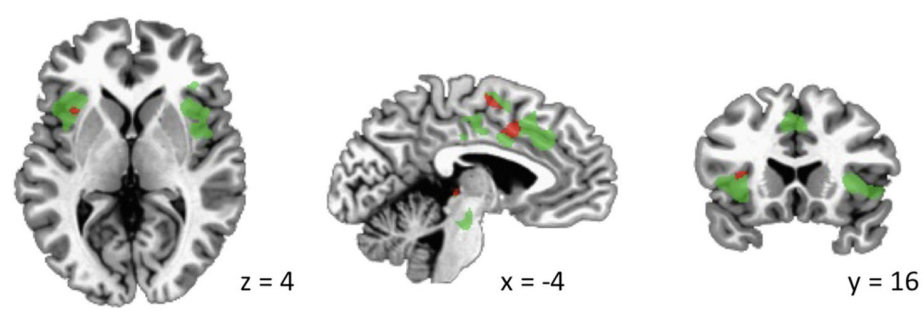

AWARE $>$ MW

MOTOR > VISUAL (motor control)

B
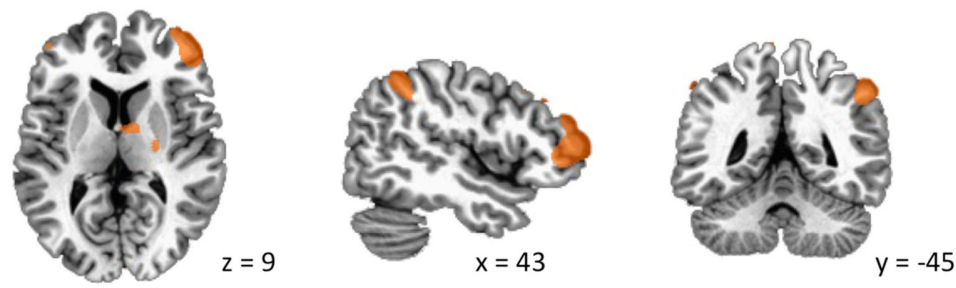

SHIFT > MW

C
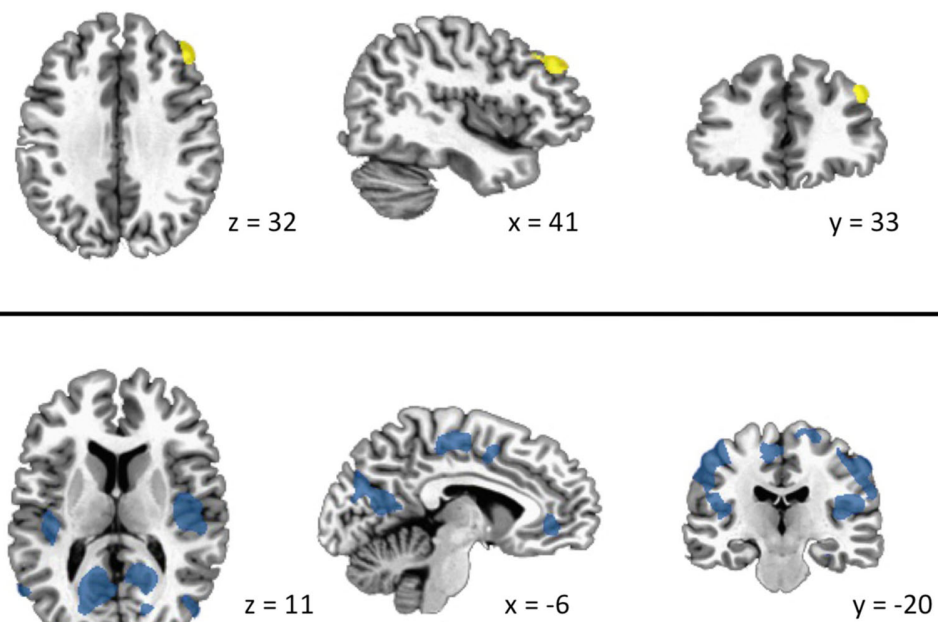

$\mathrm{MW}>\mathrm{SHIFT}$

FIGURE A2 | Significant activations for phases of interest. Specific contrasts are listed in each panel. (A) Activations during the AWARE phase are in green (due to highly robust activations, this contrast was thresholded to $p<5.0 \times 10^{-6}$ ). As the button press occurred during this phase, voxels that were also significantly active during a motor control task (motor > visual; $p<0.005)$ are shown in red. Prominent activity was detected in dorsal ACC and frontoinsular cortex. (B) Activations in lateral PFC and posterior parietal regions during the SHIFT phase. (C) Activation in dorsolateral PFC during the FOCUS phase. (D) Activations during MW phase included elements of the default mode network, as well as sensory and motor cortices and posterior insula. This figure was adapted from a previous publication (Hasenkamp et al., 2012). 


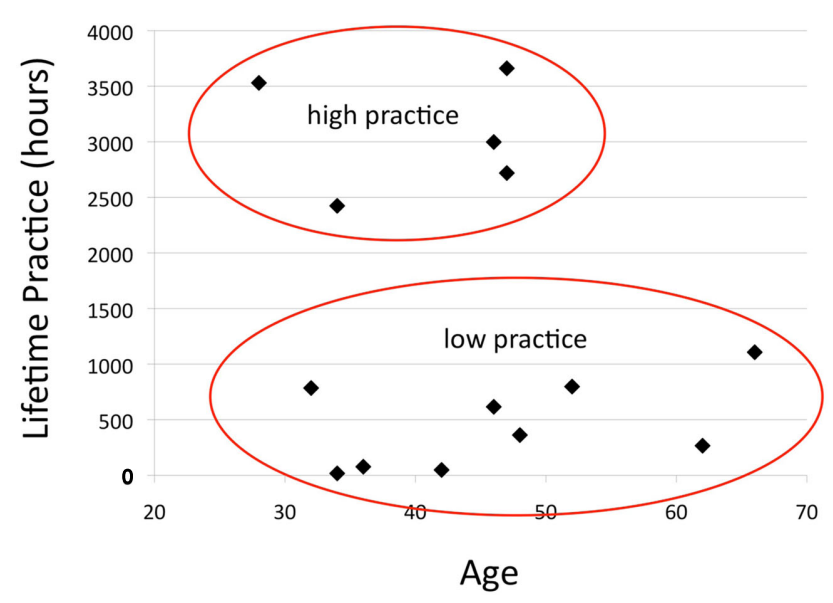

FIGURE A3 | Bimodal distribution of practice time. Each subject's estimated lifetime practice hours is plotted against his/her age. This subject population naturally fell into a bimodal distribution, with five participants having $>2000$ h of experience ("high practice") and nine participants having $<1200 \mathrm{~h}$ ("low practice"). This difference was significant by $t$-test $(p<0.001$, see Table A1).
A
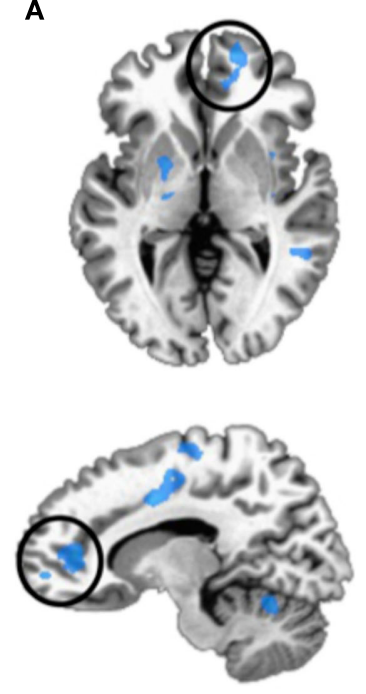

B

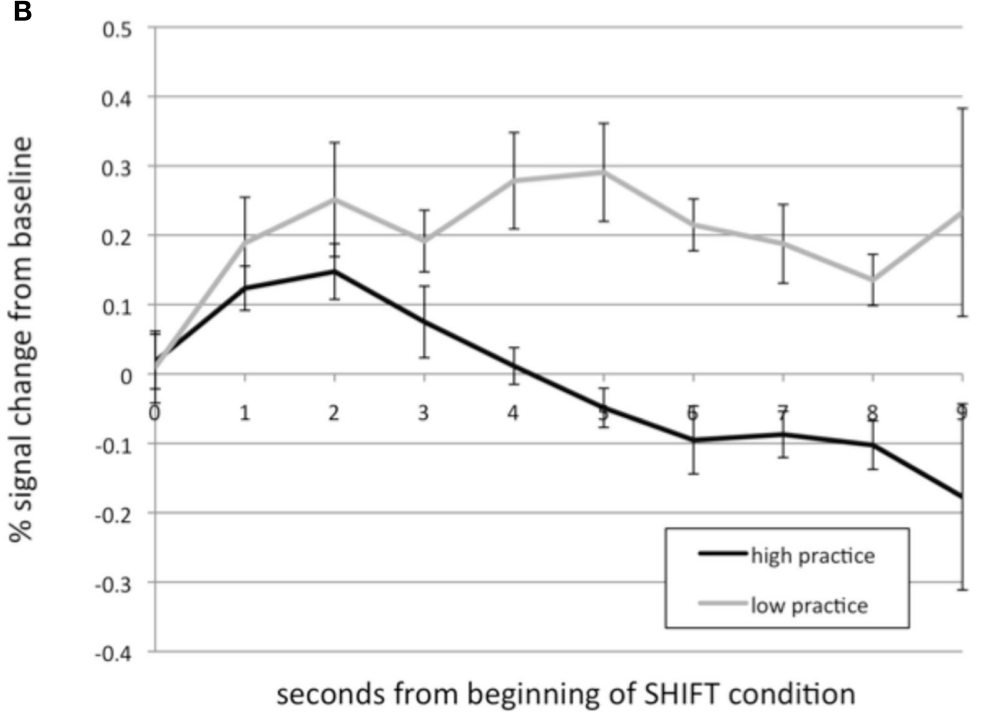

FIGURE A4 | Practice time effects. (A) Several clusters that were negatively correlated with practice time during the SHIFT phase. The medial PFC/ACC cluster that was examined in $B$ is circled. (B) Time courses from the medial PFC/ACC cluster were extracted, and HRFs were calculated from the onset of the SHIFT phase for each subject. Percent signal change (from MW, mean \pm SEM) over time is plotted for high $(N=5)$ and low $(N=9)$ practice participants. The BOLD response is significantly reduced in high practice compared to low practice participants across the modeled time series. * Main effect of group over time by repeated-measures ANOVA, $p=0.010$. This figure was adapted from a previous publication (Hasenkamp et al., 2012). 
Table A1 | Demographic information.

\begin{tabular}{llll}
\hline & $\begin{array}{l}\text { High practice } \\
(\boldsymbol{n = 5 )}\end{array}$ & $\begin{array}{l}\text { Low practice } \\
(\boldsymbol{n}=\mathbf{9})\end{array}$ & $\begin{array}{l}\text { Between groups } \\
(\boldsymbol{p} \text {-Value) }\end{array}$ \\
\hline Age (years, mean \pm SD) & $40.4 \pm 8.8$ & $46.4 \pm 12.0$ & 0.35 \\
Sex (percentage female) & 80 & 78 & 0.92 \\
Practice time (hours, mean \pm SD) & $3066.4 \pm 525.0$ & $452.9 \pm 390.9$ & $<0.001$ \\
FFMO (total score, mean \pm SD) & $149.8 \pm 11.9$ & $144.4 \pm 7.5$ & 0.32 \\
RSQ (total score, mean \pm SD) & $37.5 \pm 11.7$ & $35.7 \pm 7.7$ & 0.76 \\
Calgary SOSI (total score, mean \pm SD) & $20.8 \pm 6.4$ & $34.6 \pm 16.0$ & 0.04 \\
PANAS positive (subscore, mean \pm SD) & $32.6 \pm 1.8$ & $33.9 \pm 4.7$ & 0.48 \\
PANAS negative (subscore, mean \pm SD) & $16.0 \pm 4.6$ & $16.6 \pm 3.6$ & 0.81 \\
\hline
\end{tabular}

FFMQ, Five Factor Mindfulness Questionnaire; RSQ, Response Styles Questionnaire; Calgary SOSI, Calgary symptoms of stress inventory; PANAS, positive and negative affect scale. ${ }^{a}$ Groups were compared on each variable using t-tests, except for sex, which was assessed with Chi-square analysis.

\section{REFERENCES}

Baer, R. A., Smith, G. T., Lykins, E., Button, D., Krietemeyer, J., Sauer, S., Walsh, E., Duggan, D., and Williams, J. M. (2008). Construct validity of the five facet mindfulness questionnaire in meditating and non-meditating samples. Assessment 15, 329-342.

Carlson, L. E., and Thomas, B. C. (2007). Development of the Calgary symptoms of stress inventory (C-SOSI). Int. J. Behav. Med. 14, 249-256.

Chiesa, A., and Serretti, A. (2009). Mindfulness-based stress reduction for stress management in healthy people: a review and meta-analysis. J. Altern. Complement. Med. $15,593-600$

Nolen-Hoeksema, S., and Morrow, J. (1991). A prospective study of depression and posttraumatic stress symptoms after a natural disaster: the 1989 Loma Prieta earthquake. J. Pers. Soc. Psychol. 61, 115-121.

Watson, D., and Clark, L. A. (1999). The PANAS-X: Manual for the Positive and Negative Affect Schedule - Expanded Form. Available at: http://www.psychology.uiowa.edu/Faculty/Watson/Watson.html. 